\title{
Diabetes and residual risk of coronary heart disease
}

\author{
Richard W Nesto and Premranjan P Singh
}

Many deaths from coronary heart disease (CHD) have been prevented in patients with diabetes mellitus (DM), largely by following evidence-based guidelines and broader application of primary and secondary CHD prevention strategies. Statins, beta blockers, aspirin and angiotensinconverting-enzyme inhibitors have demonstrated this effect in various clinical settings. Several large studies indicated, however, that such strategies prevent (at best) approximately a third of CHD events. Despite optimal contemporary treatment, many patients with DM remain at high residual risk of CHD. Who are these patients? What are their clinical characteristics? What are the mechanisms? Are there novel treatments?

The Scandinavian Simvastatin Survival Study ${ }^{1}$ - a secondary CHD prevention trialrandomly assigned patients for either daily simvastatin $(40 \mathrm{mg})$ or placebo. Total mortality in simvastatin-treated patients was reduced by $43 \%$ among diabetic patients compared to $29 \%$ in patients without diabetes; however, $22.9 \%$ of diabetic patients on simvastatin experienced death or fatal myocardial infarction.

The Collaborative Atorvastatin Diabetes Study ${ }^{2}$ investigated the effectiveness of atorvastatin (10mg daily) versus placebo for prevention of major cardiovascular events in 2,838 diabetic patients. Patients on atorvastatin experienced a $37 \%$ reduction in acute CHD events, coronary revascularization, or stroke; however, $9.4 \%$ of these patients had cardiac events.

The Treating to New Targets $^{3}$ investigators evaluated the effect of atorvastatin therapy on cardiovascular morbidity and mortality in patients with CHD with or without metabolic syndrome or DM. After a median follow-up of 4.9 years, there was a $29 \%$ reduction in the risk of cardiovascular events in those treated with $80 \mathrm{mg}$ atorvastatin daily. Despite this benefit, $9.5 \%$ of these patients had a major cardiovascular adverse event and $30.9 \%$ had a minor cardiovascular event.

The PROVE IT-TIMI 22 study $^{4}$ assessed the risk of recurrent myocardial events and the relationship between LDL cholesterol and C-reactive protein

Obviously,
obesity
and insulin
resistance
should be
additional
targets for
aggressive
intervention

RW Nesto is the Chairman of and PP Singh is a Fellow in the Department of Cardiovascular Medicine at Lahey Clinic Medical Center in Burlington, $M A$, and RW Nesto is an Associate Professor of Medicine at Harvard Medical School, Boston, MA, USA.

\section{Competing interests}

They authors declared they have no competing interests.

www.nature.com/clinicalpractice doi:10.1038/ncpendmet0398
(CRP) levels in statin-treated patients with acute coronary syndromes. Fewer statin-treated patients with LDL levels below $70 \mathrm{mg} / \mathrm{dl}$ had myocardial events compared with patients with LDL over $70 \mathrm{mg} / \mathrm{dl}$; however, the group with LDL below $70 \mathrm{mg} / \mathrm{dl}$ but CRP over $2.0 \mathrm{mg} / \mathrm{dl}$ remained at an increased risk of CHD. CRP above $2.0 \mathrm{mg} / \mathrm{dl}$ was associated with features of obesity and insulin resistance, such as a high BMI, low HDL cholesterol, high triglycerides, high fasting glucose, and hypertension. This study demonstrates that, despite contemporary and aggressive secondary prevention with statins, features of insulin resistance might account for most of the high residual risk of CHD. Obviously, obesity and insulin resistance should be additional targets for aggressive intervention. Weight loss and exercise is an 'age-old' treatment that accomplishes the required goals, but is hard to initiate and maintain in the majority of patients.

New pharmacologic approaches are on the horizon that offer some hope. The cannabinoid receptor 1 blocker ${ }^{5}$ rimonabant can reduce obesity and favorably influence a number of metabolic variables, including glucose tolerance, triglycerides, HDL, CRP and adiponectin. Peroxisome proliferative activated receptor $\gamma$ (PPAR $\gamma$ ) agonists (that target insulin resistance) might also offer some promise to reduce the risk of CHD in diabetic patients, as these agents demonstrate some anti-inflammatory, antithrombotic and antiatherosclerotic properties.

Despite effective existing therapies, patients with diabetes and insulin-resistance syndromes still have considerable residual risk for CHD. We must continue to emphasize lifestyle intervention to reduce $\mathrm{CHD}$, but also evaluate new pharmacologic strategies in these high-risk patients. We await more clinical trials to see if such strategies live up to our expectations.

Supplementary information, in the form of a list of references cited in this article, is available on the Nature Clinical Practice Endocrinology \& Metabolism website. 